\title{
On-chip Pseudorandom Testing for Linear and Nonlinear MEMS
}

\author{
Achraf Dhayni, Salvador Mir, Libor Rufer, and Ahcène Bounceur \\ TIMA Laboratory, 46 av. Félix Viallet, 38031 Grenoble Cedex, France. \\ \{Achraf.Dhayni, Salvador.Mir, Libor.Rufer, Ahcene.Bounceur\}@imag.fr \\ http://tima.imag.fr
}

\begin{abstract}
In this paper we study the use of pseudorandom test techniques for linear and nonlinear devices, in particular Micro Electro Mechanical Systems (MEMS). These test techniques lead to practical BuiltIn-Self-Test techniques (BIST). We will first present the pseudorandom test technique for Linear Time Invariant (LTI) systems. Next, we will illustrate and evaluate the application of these techniques for weakly nonlinear, purely nonlinear and strongly nonlinear devices.
\end{abstract}

\section{Introduction}

Although MEMS have been around since the early 80s, most research has focused on fabrication technology, design and packaging. Therefore, unlike other areas of test research, the MEMS test area is immature and not practical for mass production. Current test and characterization practices include mainly vibration and shock techniques where mechanical stimuli are generated using an off-chip input physical module. These current techniques involve considerable difficulties. For example, tests involving mechanical stimuli require precision shaking, proper alignment of devices in fixtures, and minimization of fixture resonance [1].Temperature control is needed as well for accurate testing and calibration of commercial microsensors such as accelerometers [2]. The work in [1] and [2] shows the sophistication that accompanies inertial MEMS testing when actuated using an off-chip input physical module. This was one of the main reasons to integrate on-chip the input physical module and then contemplate Built-In Self-Test for MEMS which is very practical for mass production and in-the-field monitoring.

In microsystem testing, defects and faults, test metrics, and fault simulation practices keep the same definition as for analog ICs [3]. However microsystems sustain more failure mechanisms because of micromachining, and their fault models are more sophisticated due to the multiple energy domains, the large number of basic design elements, the new technological defects and operational failures, and the enormous possible faults which turn structural testing very much device dependent. Functional testing may be more practical than structural testing. This is the reason why only functional testing is today considered during production. In some cases it is possible to apply a simple electrical test 
signal (pulse or step) to stimulate the device under test. The transducer response is next analyzed off-chip. This is not enough for performing on-chip a functional analysis that fully tests the device and that can be exploited for other tasks such as manufacturing testing [4]. Pseudorandom (PR) testing of mixed-signal circuits has been introduced in [5]. An earlier work based on pulse-like excitation and subsequent analysis of the transient response of a mixed signal circuit is presented in [6]. In [7], an algorithm for test signature generation based on sensitivity analysis is presented. However, none of these works includes a study on the circuit implementation of the BIST technique and a comparison between different Impulse Response (IR) measurement methods taking into consideration noise and nonlinear distortions. In addition, none of these previous works consider the extension to nonlinear systems.

Several authors have considered self-test techniques for MEMS, in particular for accelerometers as in [8], [9] and [10]. Dedicated mechanical beams are used to generate an electrostatic force that mimics an external acceleration. The same idea was introduced in commercial accelerometers [11]. Alternative methods of self-test stimuli generation have been considered (e.g. electrothermal stimuli in [4], [9], and [12]. All these approaches apply electrical test pulses to stimulate the device. The transducer response is next analyzed off-chip. The work in [8] suggests computer-controlled verification and calibration when a Digital Signal Processor (DSP) is available on chip. The differential BIST presented in [13] addresses some limitations of previous self-test approaches but is only applicable for structural testing of differential sensors. A similar approach is presented in [14]. In both cases, functional testing is not considered.

It is well known that the impulse response of a LTI system provides enough information about the system functional evaluation. In [15] and [16] we have proposed a complete IR-based BIST technique for linear MEMS. The Maximal Length Sequence (MLS or m-sequence) method was used for finding the IR of linear MEMS, without any consideration of nonlinear and noise distortions that can exist in the measurement circuitry. In this chapter, different IR measurement techniques are applied to a commercial MEMS accelerometer in the presence of weak nonlinearities. They are then compared according to their immunity to nonlinear and noise distortions. The pseudorandom test methods prove high suitability for BIST implementation, and good immunity to noise and nonlinear distortion. Especially the Inverse-Repeat Sequence (IRS) pseudorandom technique which is used here for the first time in analog circuit testing.

Next, the pseudorandom method is applied for the case of pure nonlinear systems. Here, a microbeam MEMS with electrothermal excitation and piezoresistive detection is used as a case study. Finally, the pseudorandom method will be generalized for testing any nonlinear system. While considering nonlinear systems, the results of the pseudorandom method will be compared with the Volterra kernel coefficients used to model nonlinear systems. 


\section{Linear pseudorandom test method}

In [15] we have described the MEMS pseudorandom test technique. The architecture of the test approach is shown in Figure 1. The LFSR (Linear Feedback Shift Register) generates a periodic two-level deterministic MLS of length $L=2^{m}-1$, where $m$ is an integer denoting the order of the sequence. A 1-bit $\mathrm{DAC}$ is used to verify the values of the two-level signal at the output of the digital circuit of the LFSR. The 1-bit DAC is necessary for generation of a low noise analog two-level signal at the input of the DUT. Without the use of the 1-bit DAC we will need to eliminate the input signal noise by performing more averages at the ouput.

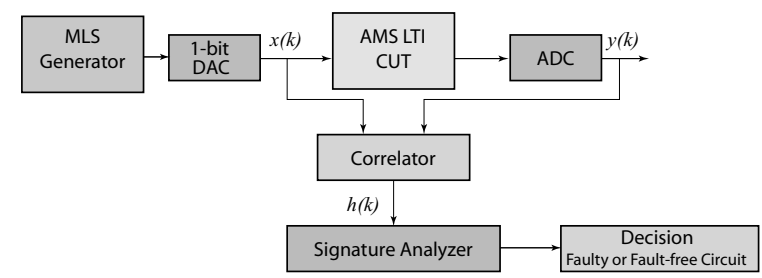

Fig. 1. Block diagram of the test approach.

The output of an LTI system is $y(k)=x(k) * h(k)$, where $\mathrm{x}(\mathrm{k})$ is the input signal and $\mathrm{h}(\mathrm{k})$ is the impulse response of the system. The input/output crosscorrelation $\phi_{x y}(k)$ can be written in terms of the convolution as:

$$
\begin{aligned}
\phi_{x y}(k) & =y(k) * x(-k) \\
& =h(k) *(x(k) * x(-k)) \\
& =h(k) * \phi_{x x}(k) \\
\Rightarrow \phi_{x y}(k) & \cong h(k) \quad \text { if } \phi_{x x}(k) \cong \delta(k)
\end{aligned}
$$

An important property of an MLS is that its autocorrelation function is, except for a small DC error, an impulse that can be represented by the Dirac delta function. We can see from Equation (1) that in the case of MLS-based measurements, crosscorrelating the system input and output sequences gives the IR. The cross-correlation operation in the case of a discrete sequence is defined by:

$$
\phi_{x y}(k)=\frac{1}{L} \sum_{j=0}^{L-1} x(j-k) y(j)
$$

Since the elements of $x(k)$ are all \pm 1 , only additions and subtractions are required to perform the multiplication in the above correlation function, which turns the design less complex and decrease the estimation period. To obtain 
the $k^{\text {th }}$ component $h(k)$ of the impulse response, we can proceed, according to Equation (2), as shown in Figure 2. Each sample of the output sequence $y(j)$ is multiplied by 1 or -1 by means of the multiplexer unit (MUX) controlled by the input sequence $x(j-k)$, and the result is added to the sum stored in the accumulator. The value obtained at the end of the calculation loop is divided by L using a shifter.

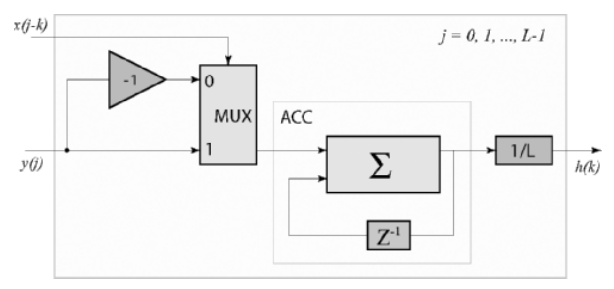

Fig. 2. Simplified Correlation Cell (SCC).

The first $m$ components of the impulse response $(h(k), k=0$ to $m-1)$ can be obtained by the scheme shown in Figure 3 .

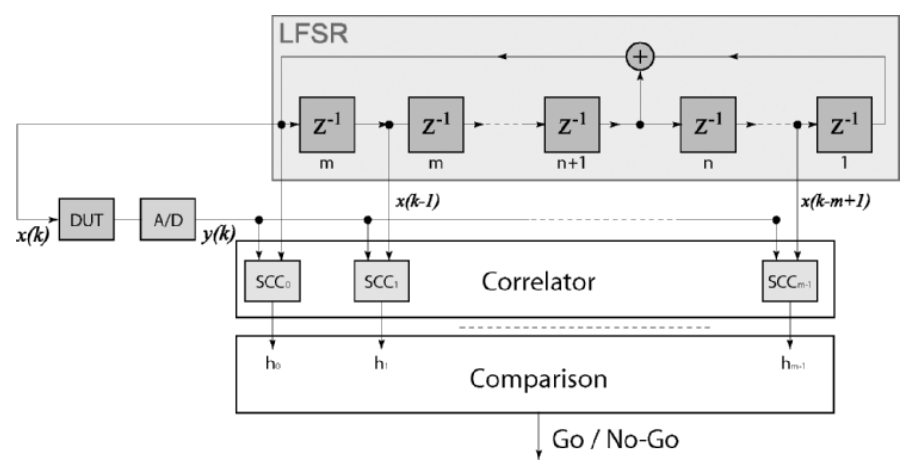

Fig. 3. BIST architecture.

The on-chip implementation shown above does not give the overall impulse response but only the first $m$ components [15],[16]. Such information can be exploited as a system pattern (test signature) that can be used for fault detection. If a larger number of components is demanded, more sophisticated algorithms can be used which would result in increased silicon overhead. In [16], we map specifications from the transfer function space to the impulse response space using Monte Carlo simulations. Then we perform a sensitivity analysis to choose the impulse response samples with highest sensitivity to faults, thus, forming the signature that permits the best fault coverage. These samples form the test signature to be compared with the tolerance range obtained by the Monte Carlo 
simulations. According with this comparison the DUT is classified as faulty or not.

\section{Case-study: MEMS accelerometer}

The measurement system in Figure 4 has been designed to stimulate the commercialized MEMS accelerometer ADXL103 [17]. The BIST circuit of Figure 3 is implemented in Labview where stimulus generation and response analysis take place.

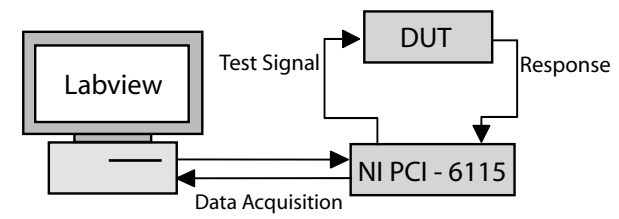

Fig. 4. Schematic representation of the measurement setup.

In Labview the PR test signal is generated and low pass filtered to eliminate the high slew rate represented by the transitions between the different levels of the PR sequence. Without the low pass filtering, the high frequency components due to high slew rates provoke nonlinear distortions and causes artifacts (spikes) in the measurements [18]. Digital low pass filtering was performed using a 5th order Kaiser-Bessel window FIR filter which is usually employed to smooth signals that contain discontinuities. In the frequency domain, this is translated by side lobe attenuation. The filtered PR signal is then applied through the data acquisition card NI PCI-6115 to the ADXL103.

The output signal is digitized in the 12-bit ADC at the input of the NI PCI-6115 card and entered to Matlab where signal processing is done to eliminate noise by averaging and calculate the impulse response components by crosscorrelating the input and output signal.

The die photo of ADXL103 sensor region is shown in Figure 5.

The block diagram of the ADXL103 measurement system is shown in Figure 6 . The activation of the digital input self-test pin (ST) by a voltage pulse induces an electrostatic force which displaces the seismic mass. The dynamic response at the output $X_{\text {out }}$ is analyzed off-chip to verify the functionality of the ADXL103.

As given by the designer [17], the transfer function of the ADXL103, for a supply voltage $\mathrm{VDD}=5 \mathrm{~V}$, is:

$$
F(s)=\frac{X_{\text {out }}}{\text { Acceleration }}=\frac{0.011}{8.374 \times 10^{-10} s^{2}+5.788 \times 10^{-6} s+1} \mathrm{mV} / \mathrm{g}
$$




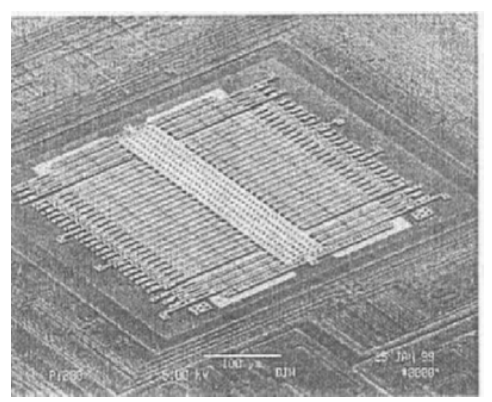

Fig. 5. Die photo of ADXL103 sensor region (4x3 self-test cells and 42 sense cells). (Source: Analog Devices; reprinted with permission.)

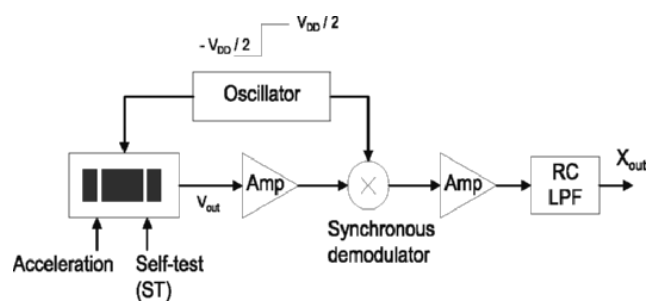

Fig. 6. Basic block diagram of the ADXL103 measurement system. (Source: Analog Devices; reprinted with permission.)

where $\mathrm{g}$ is the unit of acceleration at the input of the accelerometer $(1 g \simeq 9.8 \mathrm{~m} / \mathrm{s})$, and $X_{\text {out }}$ is the output voltage. According to Equation (3), the theoretical impulse and frequency responses of the ADXL103 are as shown in Figure 7.

For the length of the LFSR and the value of the sampling frequency, we must consider two main conditions. Firstly, if an m-sequence is mapped to an analog time-varying waveform, by mapping each binary ' 0 ' to '- 1 ' and each binary ' 1 ' to ' +1 ', then the autocorrelation function will be as shown in Figure 8. Unity for zero delay and $-1 /(2 m-1)$ for any delay greater that one sample. We can notice that for a long MLS at small $T_{c}$ (sampling period) the autocorrelation is almost an impulse function of period $=L T_{c}$. This property is used in Equation (1) to prove that the IR of a DUT equals the input/output crosscorrelation when the test signal is an MLS. According to this MLS property, the value of the multiplication of the length of the sequence by the sampling period must be greater than the time needed by the impulse response to decay to zero. Otherwise we will have impulse response aliasing. For our case, the length of the sequence is $2^{12}-1=4095$, the sampling period is $10^{-5} \mathrm{sec}$, and the decay time is approximately $1.5 \mathrm{~ms}$ (this can be observed on Figure $7(\mathrm{a})$ ). So, the first condition is satisfied since $4095 \times 10^{-5}=40.95 \mathrm{~ms}$ is greater that $1.5 \mathrm{~ms}$. 


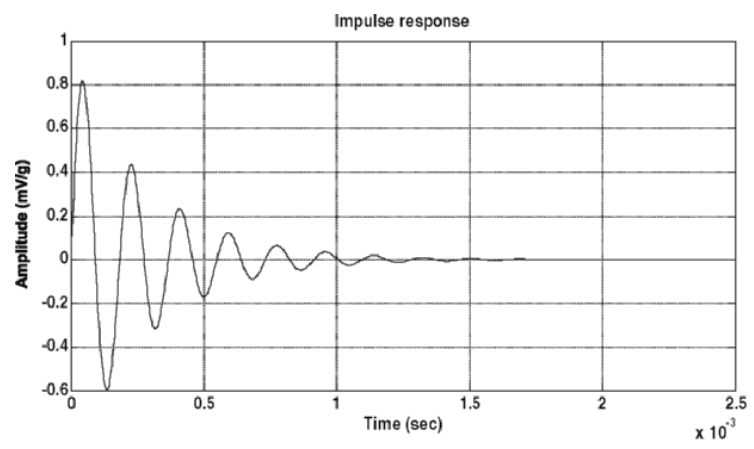

(a)

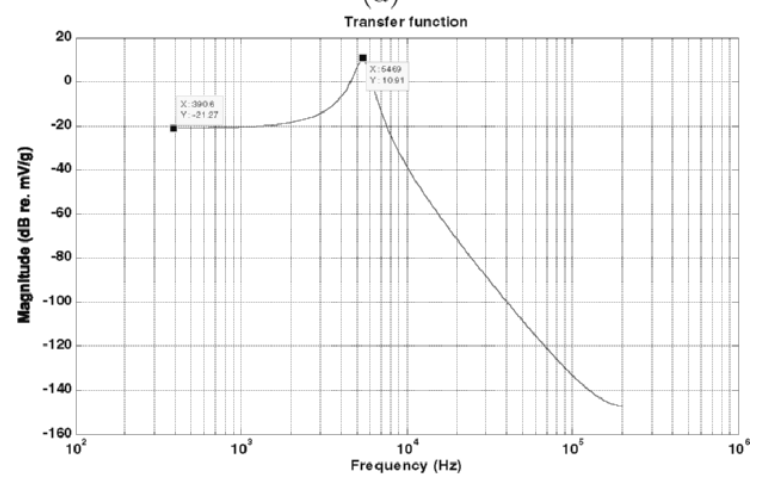

(b)

Fig. 7. (a) Impulse response, (b) frequency response of the ADXL103 model.

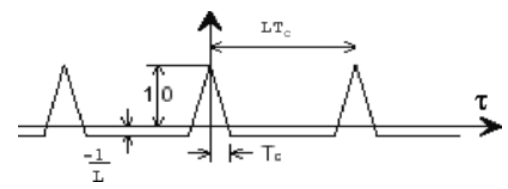

Fig. 8. Autocorrelation of a maximal length sequence represented by 1 and -1 .

Secondly, the power spectrum of the MLS is a discrete spectrum whose upper $3 \mathrm{~dB}$ roll-off frequency is about $0.45 f_{c}$. By adjusting the clock frequency, a broadband signal over a wide frequency range can be generated. According to this MLS property, the value of the sampling frequency must be chosen such that $0.45 f_{c}$ is greater than the bandwidth of the DUT. Otherwise the spectrum of the MLS will not be flat in the bandwidth of the DUT, which means that the MLS cannot be considered as a pseudorandom noise with respect to the DUT. In other words, the sampling frequency is not large enough (i.e. the sampling period $T_{c}$ is not small enough) to approximate the MLS autocorrelation function (Figure 8) to an impulse train. In our case, the sampling 
frequency is $100 \mathrm{kHz}$ and the bandwidth of the accelerometer is less than $10 \mathrm{kHz}$ (this can be observed in Figure $7(\mathrm{~b})$ ). So the second condition is satisfied since $0.45 \times 100 \mathrm{kHz}>10 \mathrm{kHz}$. It is better to choose a very high sampling frequency to avoid spectrum aliasing. However, for a certain LFSR length, the sampling frequency has an upper limit restricted by the first condition. Figure 9 shows experimental results of the application of the PR technique. Here, the impulse and frequency responses are unitless because both the input stimulus (at the SelfTest pin) and the output response are electric and of the same units (V). Using the measurement setup of Figure 4, a 12-bit LFSR and a sampling frequency of $100 \mathrm{kHz}$ (much larger than the bandwidth of the ADXL103) are programmed by Labview to generate an MLS stimulus at $\pm 5 \mathrm{~V}$ (the dynamic range of the accelerometer when stimulated through its Self-Test input). The analog output of the ADXL103 is then digitized by the 12-bit ADC of the data acquisition card. Notice that this ADC plays the role of the ADC of the PR BIST in Figure 1. Finally the IR is calculated as the input/output crosscorrelation.
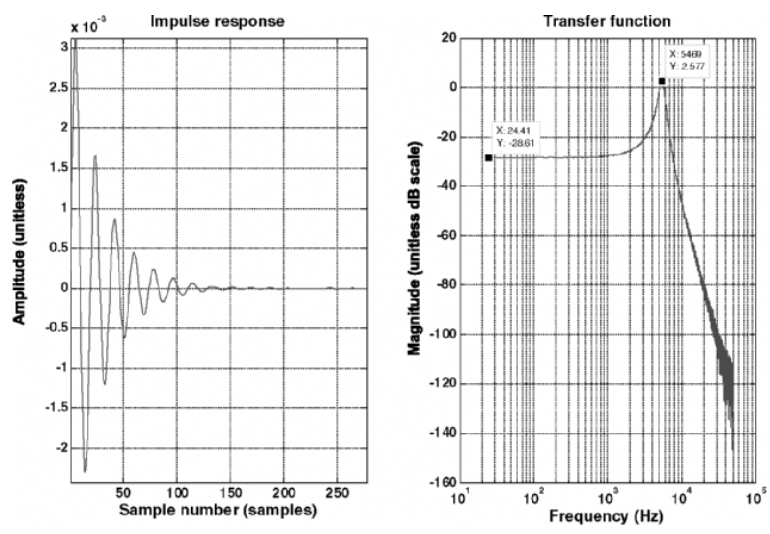

Fig. 9. Impulse and frequency responses of the ADXL103 circuit using the pseudo random impulse measurement method.

Figure 9 shows the IR and TF after 10 averages of the output signal. Averaging is used to eliminate noise. To realize 10 averages, a stimulation time of $11\left(4095 \times 10^{-5}\right)=0.45 \mathrm{sec}$ is needed. The multiplication of the MLS period by 11 rather than 10 is because we always use the first MLS to stabilize the accelerometer, and thus the measurement starts from the second sequence.

In fact, the impulse and frequency responses of the accelerometer when stimulated mechanically are highly correlated with the impulse and frequency responses when it is stimulated electrically through its self-test input. This is because the two responses represent the reaction of the same structure to a moving force, whether this force is mechanical or electrostatic. Due to this high 
correlation, the impulse and frequency responses that we have measured by stimulating the accelerometer electrically are relevant to characterization.

The gain difference between the impulse responses of Figure 7 and Figure 9 is due to the fact that the ADXL103 has lower sensitivity when stimulated artificially (at its Self-Test input pin). This difference can be calibrated. However we can notice by comparing Figure 7 and Figure 9 that using the PR BIST we can evaluate a precise impulse response. This precision is demonstrated through the transfer function (Figure 9) which shows the same resonant frequency, quality factor, bandwidth, and roll-off factor.

Notice that the IR of Figure 9 is in fact composed of 4095 samples since it is the output of the crosscorrelation operation between the 4095-sample MLS and its corresponding digitized output (in Figure 9, the IR is just zoomed in to 275 samples). To calculate the 4095-sample impulse response according to the PR BIST implementation of Figure 3, we would need to have 4095 SCCs and flip-flops. Moreover, in a BIST environment, it is too complex to implement a comparator that verifies the values of 4095 samples, each with 12 bits precision (the precision of the ADC of the data acquisition card). All this may increase the test overhead to an unacceptable value.

But in fact, only several highly fault sensitive samples (test signature) are necessary to be calculated by the BIST. A similar study to that we have presented in [16] can be applied using Monte Carlo simulations to form the test signature after a sensitivity analysis. In this way we can first derive the test signature tolerance ranges out of the specification tolerance ranges. Then, we can inject parametric variations to calculate the test metrics [3]. Finally, we can optimize the length of the MLS stimulus and the bit-precision of the BIST digital circuit.

\section{Weakly nonlinear systems}

In real life, there exist always some sources of nonlinear distortion. Here, the term "weakly nonlinear system" is used. The sources of nonlinear distortion can be due to MEMS nonidealities, due the presence of an ADC that normally has harmonic and intermodulation nonlinear distortions, and due to distortion in the analog part of the measurement circuit. Different IR measurement techniques are more or less affected by distortion according to the test signal and signal processing algorithms they use. In Section 4.1 we list different IR measurement techniques that are compared in Section 4.4 according to their signal to noise ratio SNR and distortion immunity $I_{d}$ described in Section 4.2. In Section 4.3 we describe the IRS pseudorandom test technique.

\subsection{Measurement techniques}

Theoretically, the IR of a DUT is simply the output that corresponds to a stimulus equal to a Dirac delta function $\delta(t)$. However, this is not practical 
since $\delta(t)$ is a mathematical function that can not be generated physically. Even if it is approximately generated, its high amplitude drives the circuit to work outside its dynamic range and its short duration leads to a low signal to noise ratio. Several techniques have been proposed to measure the IR response using signal processing. These can be classified into four classes:

- White Noise technique where the stimulus is a white noise and the IR is calculated by finding the DUT input/output crosscorrelation.

- Time-delay Spectrometry (TDS) [19] like the linear sine sweep and the logarithmic sine sweep [20] methods. In the linear sine sweep the IR is usually calculated by the inverse Fourier transform of the output signal. In the logarithmic sine sweep it is usually calculated by the deconvolution of the output with respect to the input using an inverse filter.

- Pulse Excitation (PE) technique which uses a single short duration pulse excitation signal, the IR is directly the corresponding output of the DUT.

- Pseudo Random (PR) technique. The test excitation signal is a pseudo random white noise like the MLS and the IRS. The IR is then found using the input/output crosscorrelation.

Among the above four techniques the $\mathrm{PE}$ and the PR are the most suitable for BIST implementation. In PE, the pulse signal generator can be implemented easily and no calculation is needed to find the IR. The problem of this method is its low SNR resulting from the low energy of the exciting signal. Averaging the output signal can be a solution for improving the SNR. In PR, the test signal (MLS or IRS) generator can be implemented easily using an LFSR, and the input/output correlation can be simply implemented using SCC. However, this is not the case of the white noise technique where the input/output crosscorrelation needs hardware to carry out all the multiplication operations. This is why it is less suitable for a BIST implementation. TDS techniques are less suitable for BIST implementation because of the complexity of the sine sweep generator and of the inverse Fourier transform calculator [19] or the inverse filter needed to perform the deconvolution of the output signal with respect to the input [20].

\subsection{Distortion immunity}

Any weakly nonlinear system can be modeled by the nonlinear model used by [21] and shown in Figure 10.

The distortion error component $e(k)$ can be calculated by subtracting the ideal IR $h(k)$ from the distorted one $h_{d}(k)$. A memoryless $r^{t h}-$ order nonlinearity $d\{$.$\} can be written as:$

$$
d\left\{x_{f}(k)\right\}=A_{d}\left[x_{f}(k)\right]^{r}
$$

where $A_{d}$ sets the amplitude of the nonlinearity. 


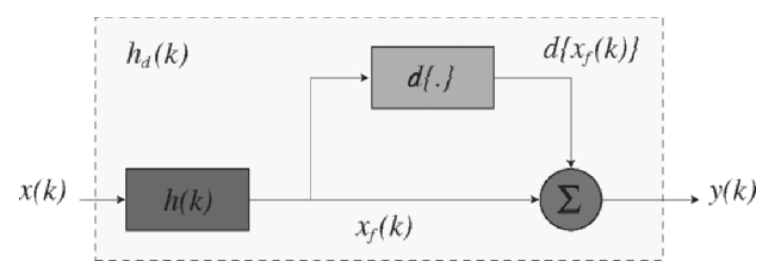

Fig. 10. Nonlinear system modeling.

In general the error due to nonlinearity contains a linear component $e_{l}(k)$ identical in shape to the linear impulse response of the system, and a nonlinear component $e_{n l}(k)$. It is the nonlinear component $e_{n l}(k)$ which causes the distortion. The linear component $e_{l}(k)$ represents only a gain error $g$ in the measurement. $e_{n l}(k)$ can be extracted from $e(k)$ according to the following equation:

$$
e_{n l}=e(k)-g \cdot h(k)
$$

$e_{n l}$ is minimized by setting the gain error $g$ to

$$
g=\frac{\sum_{k=0}^{L-1} e(k) h(k)}{\sum_{k=0}^{L-1} h^{2}(k)}
$$

where $L$ is the number of samples of IR, $\sum_{k=0}^{L-1} e(k) h(k)$ represents the energy correlated between $e(k)$ and $h(k)$, and $\sum_{k=0}^{L-1} h^{2}(k)$ is the total energy of $h(k)$. The distortion immunity $I_{d}$ of the impulse response measurement is then calculated as the ratio of the linear impulse response energy to nonlinear error energy [21] as follows:

$$
I_{d}=10 \log _{10}\left[\frac{\sum_{k=0}^{L-1} h^{2}(k)}{\sum_{k=0}^{L-1} e_{n l}^{2}(k)}\right]
$$

Distortion immunity is an important performance parameter for evaluating an IR measurement technique. But measurement environments suffer both nonlinear distortion and noise. So immunity to noise must be considered as well. In Section 4.4, the distortion and noise immunities are evaluated for each method. Finally, the best method is the one having the best immunity to distortion and noise.

\subsection{Inverse-Repeat Sequence technique}

Consider a periodic binary signal $x(k)$ suitable for impulse response measurement, where the second half of the sequence is the exact inverse of the first half, that is:

$$
x(k+L)=-x(k)
$$


The period of $2 L$ of such a sequence will always contain an even number of samples. It is proved in [21] that all even-order autocorrelations ( $r$ even) are exactly zero. Such a sequence therefore possesses complete immunity to evenorder nonlinearity after cross correlation. Due to the anti-symmetry in $x(k)$ the first order autocorrelation will also possess anti-symmetry about $L$, that is, $\phi_{1}(k)=-\phi_{1}(k+L)$. A signal that satisfies these conditions is the so-called Inverse-Repeat Sequence (IRS), obtained from two periods of MLS $s(k)$ such that the next period is inverted.

$$
\begin{aligned}
x(k) & =s(k) & \mathrm{n} \text { even, } 0 \leq k<2 L \\
& =-s(k) & \mathrm{n} \text { odd, } 0 \leq k<2 L
\end{aligned}
$$

where $L$ is the period of the generating MLS (Note that the IRS period is $2 L$ which doubles the test time). The first-order autocorrelation of an IRS ( $\left.\phi_{I R S}\right)$ is related to the corresponding signal for the generating MLS by the following expression.

$$
\begin{aligned}
\phi_{I R S}(k) & =\frac{1}{2(L+1)} \sum_{n=0}^{2 L-1} x(k) x(k+n) \\
& =\phi_{M L S}(k), \mathrm{k} \text { even } \\
& =-\phi_{M L S}(k), \mathrm{k} \text { odd } \\
& =\delta(k)-\frac{(-1)^{k}}{L+1}-\delta(k-L), \quad 0 \leq k<2 L
\end{aligned}
$$

clearly showing anti-symmetry about $L$.

By exciting a linear system with an IRS we obtain the impulse response of the system in the same way that we would if using an MLS excitation. The IRS is generated using an LFSR, and since it is a 2-level sequence the input/output crosscorrelation can be done using the SCC blocks. So the same BIST as the MLS can be used for the IRS technique.

\subsection{Comparison between PE and PR techniques}

For each of PE, MLS and IRS techniques we have used the model of Figure 10 to calculate the error signal $e(k)$. Once $e(k)$ is found, the distortion immunity $I_{d}$ can be calculated using Equations (5), (6) and (7). Table 1 shows distortion immunities of each of the three techniques for distortion orders from 2 to 5. The amplitude of the excitation signal is $20 \mathrm{dBmV}$ and that of distortion is $A_{d}=-20 \mathrm{dBm} V$. The commercial MEMS accelerometer ADXL105 from Analog Devices is taken as a DUT. 
The last two columns of Table 1 show that IRS has total immunity advantage over both MLS and PE, and MLS has total immunity advantage over PE. Notice that for even-order nonlinearities IRS has a very high immunity advantage over MLS (235.6 $d B$ at the second-order nonlinearity and $79.3 d B$ at the fourthorder nonlinearity). However only approximately $3 d B$ of immunity advantage can be offered by the IRS for the case of odd-order nonlinearity. So, the IRS appears more interesting when testing a DUT with even-order nonlinearities. However, in the presence of just odd-order nonlinearity, choosing the MLS is better because it is simpler, and the $3 d B$ of immunity advantage offered by the IRS can be compensated by a single averaging of the output sequence in the case of an MLS input. The presence of only odd-order nonlinearities is typical of systems that have odd symmetry, such as "differential" or "balanced" systems.

Table 1. Comparison between the PE, MLS and IRS test techniques.

\begin{tabular}{|c|c|c|c|c|c|}
\hline $\begin{array}{c}\text { Distortion } \\
\text { order } \\
\mathrm{r}\end{array}$ & \multicolumn{3}{|c|}{$\begin{array}{c}\text { Distortion immunity } \\
(\mathrm{dB})\end{array}$} & \multicolumn{2}{c|}{$\begin{array}{c}\text { Noise } \\
\text { and } \\
\text { distortion immunity } \\
\text { advantage of }\end{array}$} \\
\cline { 2 - 6 } & $I_{d}(P E)$ & $I_{d}(M L S)$ & $I_{d}(I R S)$ & $M L$ Sover $P E$ & IRSover $M L S$ \\
\hline 2 & 41.4 & 16.1 & 248.7 & 7.7 & 235.6 \\
\hline 3 & 63.9 & 22.1 & 23.3 & 12.1 & 3.6 \\
\hline 4 & 86.4 & 22.6 & 251.7 & 11.84 & 79.3 \\
\hline 5 & 109.7 & 25.1 & 28.1 & 11.9 & 3.7 \\
\hline
\end{tabular}

\section{Purely nonlinear systems}

In general, purely nonlinear systems can be modeled by the Hammerstein model shown in Figure 11. The term "purely nonlinear" stands for the absence of any linear behavior. This is caused by the nonlinear function at the input of the dynamic linear block.

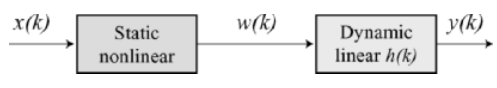

Fig. 11. Hammerstein model.

As case study of a purely nonlinear system, we consider a basic cantilever MEMS with electrothermal stimulation and piezoresistive detection. Figure 12 shows the image of a chip containing three microbeams that have been fabricated in a $0.8 \mu \mathrm{m}$ CMOS bulk micromachining technology. The surface of each cantilever is covered with heating resistors made of polysilicon. The heating of 
the cantilever causes it to bend, and the actual deflection is measured by means of piezoresistors placed at the anchor side of the cantilevers. For each cantilever, a Wheatstone bridge is used for measurement.

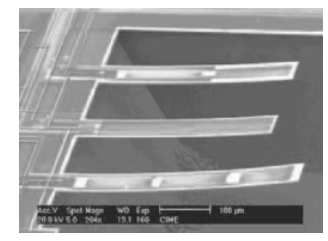

Fig. 12. Image of a fabricated microstructure.

The average temperature $T_{m}$ of the MEMS structure depends on the injected thermal power $P_{t h}$ that is a function of the voltage $V_{i}$ applied on the heating resistance $R_{h}$ according to:

$$
P_{t h}=\frac{V_{i}^{2}}{R_{h}}
$$

In this application the presence of an electrothermal coupling makes the circuit purely nonlinear. This is because of the squaring function at the input of the model, represented by Equation (11). The nonlinearity is thus static and of $2^{\text {nd }}$ order. According to Hammerstein model, the dynamic linear part is the linear IR of the suspended microbeam, and the static nonlinear part corresponds to the squaring function induced by electrothermal excitation. The pseudorandom test introduced in Section 2 is not applicable for a purely $2^{\text {nd }}$ order nonlinear system. For example, if we stimulate the microbeam by an MLS with 1 and -1 levels, $M L S_{(1,-1)}$, the sequence will be squared by the electrothermal excitation squaring function resulting in a DC signal at the input of the linear part. Of course, a DC signal is not sufficient to stimulate a linear system with memory.

To avoid the effect of squaring, a modified binary MLS with 0 and 1 levels, $M L S_{(0,1)}$, can be used. Its autocorrelation can be deduced from that of $M L S_{(1,-1)}$ according to the following:

$$
\begin{gathered}
M L S_{(0,1)}(k)=\left(M L S_{(1,-1)}(k)+1\right) / 2 \text { for } k=[0, L-1] \\
\Rightarrow \phi_{(0,1)}(k)=\frac{\phi_{(1,-1)}(k)}{4}+\frac{L-k}{4 L} \approx \frac{\delta(k)}{4}+\frac{L-k}{4 L}
\end{gathered}
$$

For $x=M L S_{(0,1)}$ and $k=[0, L-1]$, if we substitute Equation (12) in Equation (1) we obtain: 


$$
\begin{aligned}
\phi_{x y}(k) & =h(k) *\left[\frac{\delta(k)}{4}+\frac{L-k}{4 L}\right] \\
& =\frac{h(k)}{4}+\frac{1}{4} \sum_{i=0}^{k} h(i)-\frac{1}{4 L} \sum_{i=0}^{k} h(i)(k-i)
\end{aligned}
$$

Equation (13) shows how $h(k)$ can be extracted out of $\phi_{x y}(k)$ when an $M L S_{(0,1)}$ is used. This also means that $\phi_{x y}(k)$ and $h(k)$ are highly correlated which permits to form the signature in the crosscorrelation space rather than the impulse response space. This modification can be generalized. According to the Hammerstein model in Figure 11, once $x(k)$ is chosen such that $x(k)=w(k)$, the crosscorrelation of $x(k)$ and $y(k)$ can be derived as function of $h(k)$ as in Equation (13). In the case of the microbeam used in our case study, $h(k)$ is the IR of the linear part of its model. The linear part corresponds to the microbeam without considering an electrothermal excitation. Figure 13 shows the calculated impulse response $h(k)$ of the microbeam using Equation (13). Notice the resemblance between $h(k)$ and the diagonal of the $2^{\text {nd }}$ Volterra kernel in Figure 14. Volterra kernels are functions used to model nonlinear systems [22] and we will use them in the next Section.

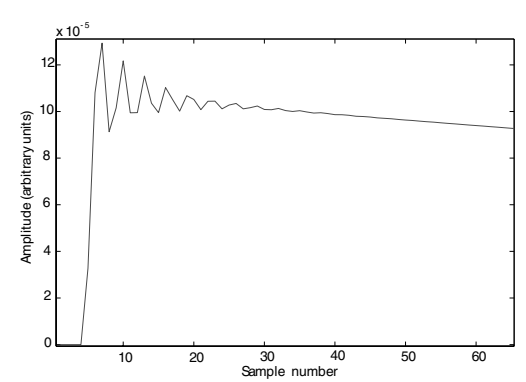

Fig. 13. IR of the microbeam.

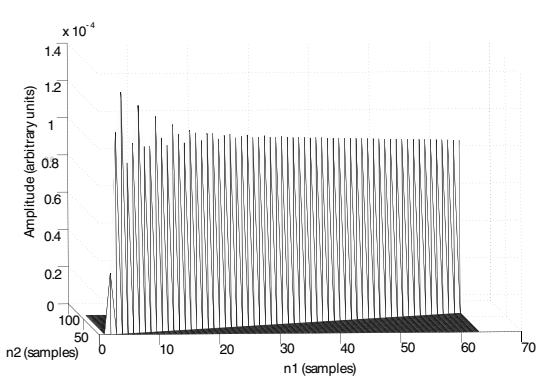

Fig. 14. $2^{\text {nd }}$ Volterra kernels of the microbeam. 
Therefore, for MEMS that can be modeled by the Hammerstein model, there is no need of sophisticated nonlinear modeling since the same results can be obtained with a simple modification of the test signal in the proposed BIST.

\section{Strongly nonlinear systems}

Here we consider the nonlinear systems that cannot be modeled according to the simple Hammerstein model as the case of purely nonlinear systems. In our work we make use of the Volterra modeling technique to test strongly nonlinear devices. It has been shown in [22] that any time-invariant nonlinear system with fading memory can be approximated by a finite Volterra series to an arbitrary precision according to the following equation:

$$
y(k)=h_{0}+\sum_{r=1}^{N} \sum_{m_{1}=0}^{M-1} \cdots \sum_{m_{r}=0}^{M-1} h_{r}\left(m_{1}, \cdots, m_{r}\right) \prod_{j=1}^{r} x\left(k-m_{j}\right)
$$

where $x$ and $y$ are respectively the input and output of the system, $N$ is the nonlinearity order, $M$ is the memory of the system, and $h_{r}\left(m_{1}, \cdots, m_{r}\right)$ represents a coefficient of the $r^{t h}-$ order Volterra kernel $h_{r}$. The kernel $h_{r}$ carries information about the $r^{t h}-$ order nonlinear behavior of the system. Our interest is to calculate the kernel coefficients of a nonlinear DUT, then compare them with the typical values to test whether a fault exists or not. Existing methods for the identification of Volterra kernels have proved computationally burdensome. In [22] the authors have proposed an efficient method to determine the Volterra kernels, where they make use of the Wiener general model in Figure 15.

According to this method, the system is stimulated by a multilevel MLS (Figure 16) to extract the Wiener coefficients from the values of the sampled output response. The advantage of this method is that the multilevel MLS stimulus can be easily generated on-chip. The Volterra kernels are then obtained from the Wiener model using a simple calculation.

To illustrate the physical meaning of Volterra kernels, let us consider the block models shown in Figure 17. Figure 18 shows the $1^{\text {st }}$ and $2^{\text {nd }}$ kernels of each of these models, calculated by the algorithm that we have implemented based on the technique explained in [22].

The first two kernels of the linear system in Figure 17(a) are shown in Figure 18(a) and Figure 18(b) respectively. Notice how the $1^{\text {st }}$ kernel represents the linear impulse response and the $2^{n d}$ kernel is equal to zero since the system is linear. The first two kernels of the nonlinear system of Figure 17(b) are shown in Figure 18(a) and Figure 18(c) where the $2^{\text {nd }}$ kernel is not equal to zero anymore. The $1^{\text {st }}$ kernel is always the same because the linear part of the systems in Figure 17(a) and Figure 17(b) is the same. Similarly, the system of Figure $17(\mathrm{c})$ has the same $1^{\text {st }}$ kernel and the $2^{\text {nd }}$ kernel is shown in Figure 18(d). 


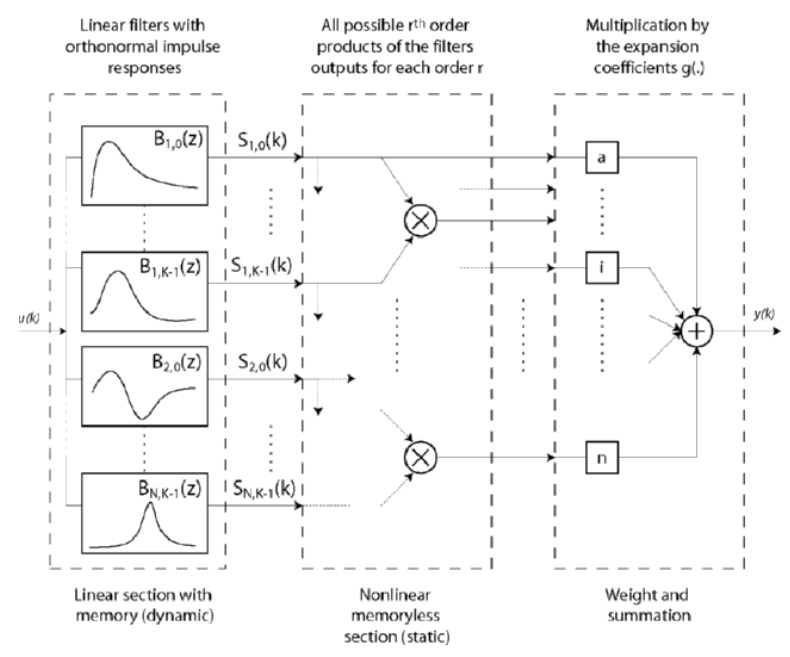

Fig. 15. Wiener model with orthonormal basis.

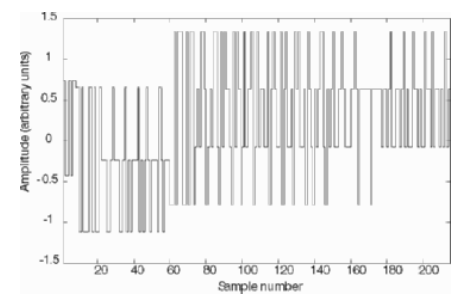

Fig. 16. Multilevel MLS stimulus.

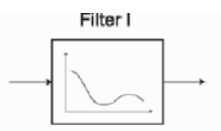

(a)

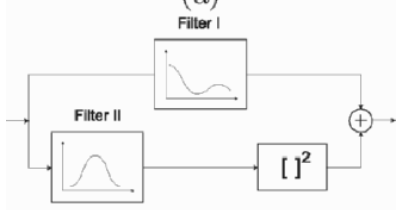

(b)

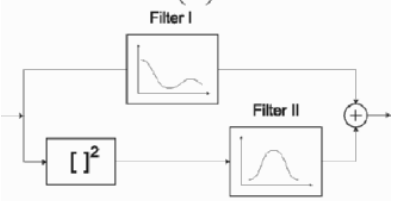

(c)

Fig. 17. (a) linear system, (b) and (c) $2^{\text {nd }}$ order nonlinear systems. 

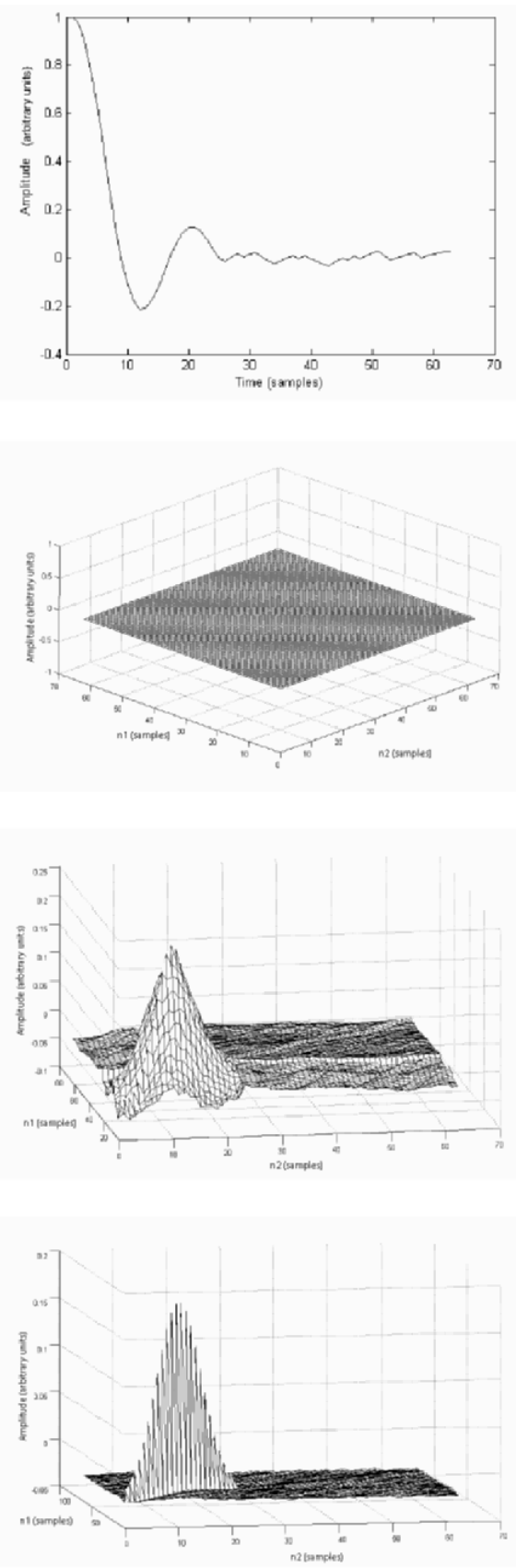

(d)

Fig. 18. Volterra kernels of the systems in Figure 17: (a) $1^{\text {st }}$ kernel for all systems, (b), (c) and (d) $2^{\text {nd }}$ kernels for the systems in Figures $17(\mathrm{a}), 17(\mathrm{~b})$ and $17(\mathrm{c})$, respectively. 
After finding Volterra kernels we can extract design properties out of these kernels and prove that they correspond really to the system. For example, the $1^{\text {st }}$ kernel in Figure 18(a) is nothing but the impulse response of the FIR Filter I which plays the role of the linear part in the systems of Figure 17. This proves the correctness of the $1^{\text {st }}$ kernel. The nonlinearity of the system in Figure $17(\mathrm{c})$ is represented by squaring each input sample. Thus, there is no multiplication between different input samples at different delays, which means that all the $2^{\text {nd }}$ kernel coefficients at $n_{1} \neq n_{2}$ are zero. That is why Figure $18(\mathrm{~d})$ has values only through the diagonal $n 1=n 2$. Moreover, the values through the diagonal correspond to the impulse response of the FIR Filter II since it is in cascade with the squaring function. For the purpose of testing, we will be interested in finding a test signature composed of only several Volterra samples that are highly sensitive to faults. A similar signature analysis to that of linear MEMS [16] can be applied. Finally the signature is compared with the tolerance range to decide whether the nonlinear MEMS functions correctly or not.

\section{Validity of the binary PR BIST for testing nonlinear MEMS}

It can be proved that applying the pseudorandom test method to a nonlinear system results in:

$$
\begin{aligned}
\phi_{x y}(k)= & \sum_{i=0}^{L-1} h_{1}(i) \cdot \phi_{1}(k-i)+\sum_{i=0}^{L-1} \sum_{j=0}^{L-1} h_{2}(i, j) \cdot \phi_{2}(k-i, k-j) \\
& +\sum_{i=0}^{L-1} \sum_{j=0}^{L-1} \sum_{m=0}^{L-1} h_{3}(i, j, m) \cdot \phi_{3}(k-i, k-j, k-m)+\cdots
\end{aligned}
$$

where each term is an r-dimensional convolution of a Volterra kernel $h_{r}\left(k_{l}, k_{2}\right.$, $\left.\cdots, k_{r}\right)$ with the r-dimensional autocorrelation function of the input sequence $\phi_{r}\left(k_{l}, k_{2}, \cdots, k_{r}\right)$. The first term, $\sum_{i=0}^{L-1} h_{1}(i) \cdot \phi_{1}(k-i)$, equals $h_{1}(k)$ for the case of an $M L S$ which means that $\phi_{x y}(k)$ is directly related to $h_{1}(k)$, the linear behavior of the system. So whenever there is a fault harming the linear behavior it will be displayed in the input/output crosscorrelation space. In this case a similar Monte Carlo simulation is used to find the tolerance range in the crosscorrelation space rather than the impulse response space, and to perform a sensitivity analysis to form a test signature composed out of several highly sensitive-to-fault crosscorrelation samples.

As a result, the PR BIST is valid for any time invariant analog system and for all faults that harm the linear behavior. We do in fact suppose that most often faults that affect the nonlinear behaviors do also affect the linear behavior. The multi-level PR BIST can be used for nonlinear MEMS characterization and it is only necessary for testing a rare category of nonlinear microsystems where 
some faults can be nonlinear and only influence the nonlinear behavior. In this case, Volterra kernels can be used to test and diagnose this kind of faults. Finding Volterra kernels can also be used to classify linear and nonlinear faults which is important for fault injection and simulation. Faults that affect the linear behavior ( $1^{\text {st }}$ Volterra kernel) are linear faults, and faults that only affect the nonlinear behavior (higher order Volterra kernels) are nonlinear faults.

\section{Conclusions and further work}

This chapter has presented an evaluation of different IR measurement methods suitable for simple MEMS BIST techniques. These techniques have been applied to a commercial MEMS accelerometer. As a result, the IRS is the most suitable when even-order nonlinearities exist. We have proved that it has a very high total immunity against even-order nonlinearities. Such nonlinearities vanish for differential systems where the MLS can give the same results as the IRS. The pseudorandom test method has been modified and applied to a purely nonlinear microbeam with electrothermal excitation. The resulting input/output crosscorrelation samples are the Volterra kernel coefficients needed for modeling. Finally, the validity of pseudorandom methods for nonlinear devices has been discussed. The multi-level PR BIST can be considered as an advanced version of the PR BIST presented in Section 2 for linear MEMS. With the new version we are capable of testing and characterizing any linear or nonlinear circuit. However the new PR BIST version demands the presence of a DSP on-chip to calculate the Volterra kernels.

Finding Volterra kernels allows isolating the linear behavior from the nonlinear behavior of nonlinear systems. The linear impulse response was extracted from the total response by using multi-level pseudorandom sequences. The technique is compatible with the PR BIST that was demonstrated for linear and purely nonlinear systems. This is because we are still using pseudorandom stimuli suitable for on-chip implementation, and also because the test is again based on the measurement of the linear IR where the tolerance range and the test signature are formed. The test technique can be simplified by finding the test signature in the space of Wiener expansion coefficients rather than Volterra coefficients. According to the test signature only some necessary modified MLSs are selected to form shorter multi-level sequences. In this way, less calculation is needed to find only the several Wiener expansion coefficients that form the test signature. We consider this step as the main perspective of this work.

Finally we introduce the definition of linear and nonlinear faults and we show that the multi-level PR BIST is necessary when nonlinear faults exist. The MEMS pseudorandom BIST techniques have been studied using a real MEMS device where MLS signals were generated and analyzed using Labview and a date acquisition card. 


\section{References}

1. R.W. Beegle, R.W. Brocato, and R.W. Grant, IMEMS Accelerometer Testing - Test Laboratory Development and Usage, Proceedings of International Test Conference, September 1999, pp. 338-347.

2. T. Maudie, A. Hardt, R. Nielsen, D. Stanerson, R. Bleschke, and M. Miller, MEMS Manufacturing Testing: An Accelerometer Case Study, Proceedings of International Test Conference, September 2003, pp. 843-849.

3. S. Sunter and N. Nagi, Test metrics for analog parametric faults, Proceedings of VTS, 1999, pp. 226-234.

4. B. Charlot, S. Mir, F. Parrain, and B. Courtois, Generation of Electrically Induced Stimuli for MEMS Self-test, Journal of Electronic Testing: Theory and Applications, December 2001, vol. 17, no. 6, pp. 459-470.

5. C.Y. Pan and K.T. Cheng, Pseudorandom Testing for Mixed-signals Circuits, IEEE Transactions on Computer-Aided Design of Integrated Circuits and Systems, 1997, vol. 16, no. 10, pp. 1173-1185.

6. P.S.A. Evans, M.A. Al-Qutayri, and P.R. Shepherd, A Novel Technique for Testing Mixed-Signal ICs, Proceedings of European Test Symposium, 1991, pp. 301306.

7. F. Corsi, C. Marzocca, and G. Matarrese, Defining a BIST-oriented Signature for Mixed-signal Devices, IEEE Proceedings of Southwest Symposium on MixedSignal Design, 2003, pp. 202-207.

8. H.V. Allen, S.C. Terry, and D.W. de Bruin, Self-Testable Accelerometer Systems, Proceeding of Micro Electro Mechanical Systems, 1989, pp. 113-115.

9. M. Aikele, K. Bauer,W. Ficker, F. Neubauer, U. Prechtel, J. Schalk, and H. Seidel, Resonant Accelerometer with Self-test, Sensors and Actuators A, Auguest 2001, vol. 92, no. 1-3, pp. 161-167.

10. R. Puers and S. Reyntjens, RASTAReal-Acceleration-for-Self-Test Accelerometer: A New Concept for Self-testing Accelerometers, Sensors and Actuators A, April 2002, vol. 97-98, pp. 359-368.

11. L. Zimmermann, J.P. Ebersohl, F. Le Hung, J.P. Berry, F. Baillieu, P. Rey, B. Diem, S. Renard, and P. Caillat, Airbag application: a microsystem including a silicon capacitive accelerometer, CMOS switched capacitor electronics and true self-test capability, Sensors and Actuators A, 1995, vol. A 46, no. 1-3, pp. 190-195.

12. V. Beroulle, Y. Bertrand, L. Latorre, and P. Nouet, Test and Testability of a Monolithic MEMS for Magnetic Field Sensing, Journal of Electronic Testing, Theory and Applications, October 2001, pp. 439-450.

13. N. Deb and R.D. Blanton, Built-In Self-Test of CMOS-MEMS Accelerometers, Proceedings of International Test Conference, October 2002, pp. 1075-1084.

14. X. Xiong, Y.L. Wu, and, W.B. Jone, A Dual-Mode Built-In Self-Test Technique for Capacitive MEMS Devices, Proceedings of VLSI Test Symposium, April 2004, pp. $148-153$.

15. L. Rufer, S. Mir, E. Simeu, and C. Domingues, On-chip Pseudorandom MEMS Testing, Journal of Electronic Testing: Theory and Application, 2005, pp. 233241.

16. A. Dhayni, S. Mir, and L. Rufer, MEMS Built-In-Self-Test Using MLS, IEEE Proceedings of 9th European Test Symposium, 2004, pp. 66-71.

17. http://www.analog.com/UploadedFiles/(Data_Sheets/279349530ADX $L 103_{2} 03_{0}$. $p d f$ 
18. A. Dhayni, S. Mir, L. Rufer, and A. Bounceur, Nonlinearity effects on MEMS onchip pseudorandom testing, Proceedings of International Mixed-Signals Testing Workshop, Cannes, France, June 2005, pp. 224-233.

19. S. Müller and P. Massarani, Transfer Function Measurement with Sweeps, Journal of Audio Engineering Society, 2001, vol. 49, no. 6, pp. 443-471.

20. A. Farina, Simultaneous Measurement of Impulse Response and Distortion with a Swept-sine Technique, presented at the 108th Convention of Audio Engineering Society, Journal of Audio Engineering Society, vol. 48, pp. 350, preprint 5093.

21. C. Dunn and M.O. Hawksford, Distortion Immunity of MLS-Derived Impulse Response Measurements, Journal of Audio Engineering Society, 1993, vol. 41, no. 5, pp. 314-335.

22. M. Reed and M. Hawksford, Identification of Discrete Volterra Series Using Maximum Length Sequences, IEE Proceedings on Circuits, Devices and Systems, 1996, pp. 241-248. 